\title{
Are "Unstable" Burst Fractures Really Unstable?
}

\author{
Jun Hyuk Woo, ${ }^{1}$ Hyun Woo Lee, ${ }^{2}$ Hong June Choi, ${ }^{1}$ Young Min Kwon' \\ Department of Neurosurgery, Dong-A University Hospital, Dong-A University College of Medicine, Busan, Korea \\ Department of Neurosurgery, ${ }^{2}$ Gupo Sungsim Hospital, Busan, Korea
}

Objective : The stability is an important factor to decide the treatment plan in thoracolumbar burst fracture patients. Patients with an unstable burst fracture generally need operative management. Decrease in vertebral body height, local kyphosis, involvement of posterior column, and/or canal compromise are considered important factors to determine the treatment plan. On the other hand, in thoracolumbar injury classification system (TLICS), surgery is recommended in patients with TLICS of more than 5 points. The purpose of this study was to apply the TLICS score in patients with thoracolumbar burst fractures and to distinguish the differences of treatment plan on burst fracture.

Methods : All patients, diagnosed as a thoracolumbar burst fracture between January 2006 and February 2019 were included in this study. Unstable thoracolumbar burst fracture was defined as burst fracture with neurologic deficit, three-column injury, kyphosis over 30 degrees, decrease of anterior body height over 40 percent and canal comprise more than $50 \%$. TLICS score was measured with morphology, neurological involvement and posterior ligamentous complex integrity. The existence of instability was compared with TLICS score.

Results : Total 233 patients (131 men, 102 women) were included in this study. In Denis classification, 51 patients (21.9\%) diagnosed as stable burst fracture while 182 patients (78.1\%) had unstable burst fracture. According to TLICS, 72 patients (30.9\%) scored less than 4, while 161 patients (69.1\%) scored 4 or more. All the patients with stable burst fracture scored 2 in TLICS. Twenty-one patients (9.0) scored 2 in TLICS but diagnosed as unstable burst fracture. Thirteen patients had over $40 \%$ of vertebra body compression, four patients had more than $50 \%$ of canal compromise, three patients had both body compression over $40 \%$ and kyphosis over 30 degrees, one patients had both body compression and canal compromise. Fifteen patients presented kyphosis over 30 degrees, and three (20\%) of them scored 2 in TLICS. Seventy-three patients presented vertebral body compression over $40 \%$ and 17 (23.3\%) of them scored 2 in TLICS. Fifty-three patients presented spinal canal compromise more than $50 \%$, and five (9.4\%) of them scored 2 in TLICS.

Conclusion : Although the instability of thoracolumbar burst fracture was regarded as a critical factor for operability, therapeutic strategies by TLICS do not exactly match with the concept of instability. According to the concept of TLICS, it should be reconsidered whether the unstable burst fracture truly unstable to do operation.

Key Words : Spinal injuries · Spinal fractures · Classification.

- Received : April 6, $2021 ・$ Revised : June 20, $2021 ・$ Accepted : August 25, 2021

- Address for reprints : Young Min Kwon

Department of Neurosurgery, Dong-A University Hospital, Dong-A University College of Medicine, 26 Daesingongwon-ro, Seo-Gu, Busan 49201, Korea

Tel : +82-51-240-5246, Fax : +82-51-242-6714, E-mail : ymkwon@dau.ac.kr, ORCID : https://orcid.org/0000-0002-9870-5257

This is an Open Access article distributed under the terms of the Creative Commons Attribution Non-Commercial License (http://creativecommons.org/licenses/by-nc/4.0) which permits unrestricted non-commercial use, distribution, and reproduction in any medium, provided the original work is properly cited. 


\section{INTRODUCTION}

Nearly $90 \%$ of all spinal injuries involve the thoracolumbar vertebra, and $10 \%$ to $20 \%$ of such injuries are burst fractures ${ }^{1,4,16)}$. The term "burst fracture" was first defined by Holdsworth" as a fracture result from vertical compression leading to herniation of the nucleus pulposus of the vertebral disk through the upper end plate, resulting in the disruption of the vertebra from within. The 3-column theory presented by Denis describes both the mechanism of the injury and the concept of spinal stability and the burst fracture can be 2 or 3 column injuries ${ }^{4,5)}$. According to Denis, burst fracture is described as the compression of the anterior and middle vertebral columns which causes retropulsion of a posterior vertebral body fragment into the spinal canal.

Many authors have provided important insights that helped to distinguish between different types of thoracolumbar fractures and several thoracolumbar spine injury classification systems had been introduced to help clinical and surgical treatment ${ }^{4,6,9,11,15)}$. Until Denis ${ }^{4)}$, who proposed three-column model, the assessment of the integrity of the posterior ligamentous complex was not properly considered. Denis classified spinal injury into four groups (compression fracture, burst fracture, seat-belt injury and fracture-dislocation) depending on the injury mechanism and the degree of injury. However, McAfee pointed out that Denis' thought overestimates the effect of the stability of middle column so that it promotes the unnecessary surgeries. McAfee classified thoracolumbar fracture as six types depending on compression, distraction, and direct shearing force on middle column utilizing computed tomography (CT) scan. As a result, he divided bursting fracture into two categories, stable fracture and unstable fracture, and defining unstable fracture as three column injury including posterior column injury. He also argued that neurological deficits, posterior element disruption, kyphosis above 20 degrees, loss of vertebral body height by $50 \%$ or more, and canal compromise of the free bony fragments as factors that indicate instability in rupture fractures ${ }^{11)}$. In addition, McCormack et al. ${ }^{12)}$ supplemented this to introduce loadsharing classification to influence the determination of the scope of surgical treatment.

The thoracolumbar injury classification system (TLICS) has been developed to improve injury classification and guide surgical decision-making. TLICS includes fracture morphology, neurologic status, and integrity of posterior ligamentous com-
Table 1. Summary of the revised scale, the TLICS

\begin{tabular}{ll}
\hline Component & Score \\
\hline Morphology type & 1 \\
Compression & 2 \\
$\quad$ Burst & 3 \\
Translation/rotation & 4 \\
Distraction & \\
Neurological involvement & 0 \\
Intact & 2 \\
Nerve root & \\
Cord, conus medullaris & 2 \\
$\quad$ Complete & 3 \\
Incomplete & 3 \\
Cauda equina & \\
PLC & \\
Intact & 0 \\
Injury suspected/indeterminate & 2 \\
Injured & 3 \\
\hline
\end{tabular}

TLICS : thoracolumbar injury classification and severity score, PLC: posterior ligamentous complex

plex (Table 1). Patients with TLICS score 1-3 deserve conservative treatment and patients with TLICS score more than 5 needs surgical treatment. Patients with TLICS score 4 could consider surgical or conservative treatment. The aim of this study was to identify the appropriateness of decision-making according to general concepts and TLICS.

\section{MATERIALS AND METHODS}

This study was approved by the Institutional Review Board of Dong-A University Hospital (DAUHIRB-21-194).

From January 2005 to February 2019, patients who visited a single tertiary hospital and diagnosed as burst fracture in thoracic or lumbar spine were included. Patients' simple radiographs, CT and magnetic resonance image of injured spine were reviewed. Their neurologic status were thoroughly reviewed and their treatment options were also checked.

It is difficult to define the critical values of the parameters that may be required for determining the stability of a burst fracture. In this study, we define unstable thoracolumbar burst fracture in case of injury of all three columns, severe ca- 
nal compromise more than $50 \%$ in axial CT scan of injured level, vertebral height loss more than 40\%, kyphosis more than $30^{\circ}$ in plain X-ray and neurologic deficit, which are proposed as indicators of implementing pedicle screw fixation in Korea Health Insurance Review \& Assessment Service Center. Canal compromise was calculated on the axial view of CT scan of mostly injured level. In plain X-ray lateral view, compression ratio of vertebral height was evaluated as the ratio of the fractured front vertebral body height to the mean of the front height of the adjacent upper and lower vertebral body. Kyphotic angle was measured in plain X-ray lateral view between the superior end plate of the vertebra above and the inferior vertebra below the fractured level (Fig. 1). According to the criteria of this paper, the operation was performed in consideration of the patient's general condition. Injuries were described as thoracic (T1-10), thoracolumbar (T11-L2), or lumbar (L3-5). All the data of the patients was reviewed by three neurosurgeons according to McAfee classification and TLICS system at the most severely injured level twice in another day. The parameters of unstable thoracolumbar burst fracture were also checked by three different reviewers twice. Exclusion criteria included pathologic fractures (infection, cancer) and those with chronic or age-indeterminate thoracic and lumbar traumas.

\section{RESULTS}

Total 233 patients were included in this study and 131 patients (56.2\%) were men while 102 patients( 45.8\%) were women. Their mean age was 51.9 year-old range from 15 to 83 . Standard deviation of the age was 16.3 years. Nine patients (3.9\%) had thoracic burst fracture, 171 patients $(73.4 \%)$ had burst fracture in thoracolumbar lesion (T11-L2) and 53 patients (22.7\%) had burst fracture in L3-5 level (Table 2).

Fifty-one patients (21.9\%) were diagnosed as stable thoracolumbar burst fracture while 182 patients (78.1\%) were diagnosed as unstable burst fracture. Injury of all three columns was the most common reason $(65.2 \%, 152 / 233)$ which causes unstable thoracolumbar burst fracture. According to TLICS classification, 72 patients (30.1\%) scored 2, 38 patients $(16.3 \%)$ scored 4, 39 patients (16.7\%) scored 5, 26 patients $(11.2 \%)$ scored 6, 37 patients (15.9\%) scored 7, and 21 patients $(9.0 \%)$ scored 8 . In patients group with stable burst fracture, every 51 patients scored 2 in TLICS. Patients with unstable thoracolumbar burst fracture had various TLICS score from 2 to 8 . Twenty-one patients (9.0\%) had TLICS score less than 3, 38 patients $(16.3 \%)$ had TLICS score of 4 , and 123 patients (52.8\%) scored more than 5 in TLICS (Table 3).

Among 21 patients (9.0\%) who diagnosed as unstable thoracolumbar burst fracture but presented TLICS 2 score, 17 patients (80.9\%) had more than $40 \%$ of vertebral body compres-
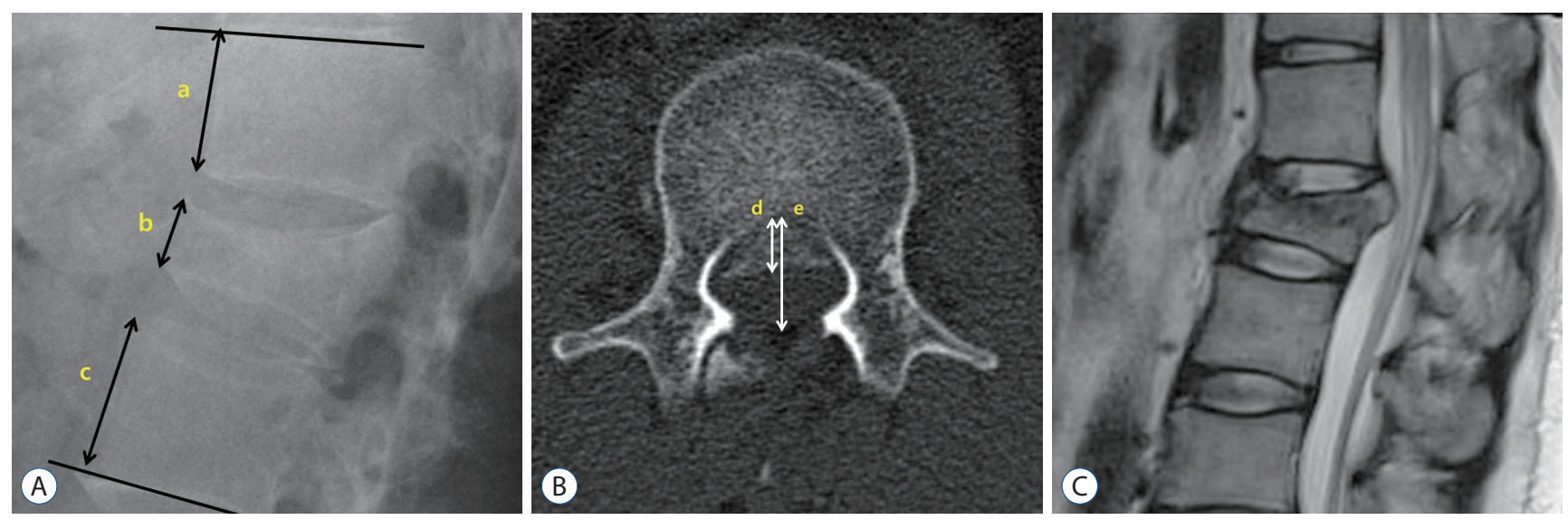

Fig. 1. Plain X-ray, computed tomography (CT) and magnetic resonance image (MRI) of a 36-year-old male patient with a L2 burst fracture without neurological deficits. Radiologic measure of parameters. In plain X-ray lateral view, compression ratio of vertebral height was calculated by [1 - 2b / $(a+c)]$, as the ratio of the fractured front vertebral body height to the mean of the adjacent upper and lower vertebral frontal body. He showed $55 \%$ of compression ratio. Kyphotic angle was measured by Cobb's angle, between the superior end plate of the vertebra above and the inferior vertebra below the fractured level. He showed $9.2^{\circ}$ of kyphosis (A). Spinal canal compromise was measured in axial image of CT scan by [1- d/e]. He showed $47 \%$ of spinal canal compromise (B). MRI of injured level present no signs of posterior ligamentous complex damage (C). His thoracolumbar injury classification system score was 2 (morphology, 2; posterior ligamentous complex, 0; neurology, 0). He underwent posterior decompression and pedicle screw fixation. 
sion ratio, two patients (9.5\%) had spinal canal compromise more than $50 \%$, one patient (4.8\%) showed both severe body compression and focal kyphosis, and one patient showed both canal compromise and body compression (Table 4). Eighteen patients (85.7\%) had unstable burst fracture in thoracolumbar (T10-L2) level while three patients (14.3\%) had unstable burst fracture in lumbar (L3-5) level. L1 vertebra (38.1\%) was the most commonly involved level in this group of patients. Fourteen patients (66.7\%) were treated with surgery while seven patients (33.3\%) had conservative treatment.

Table 2. Demographics and characteristics of patients

\begin{tabular}{lc}
\hline & Value \\
\hline Total patients & $233(100.0)$ \\
Age (years) & $51.9 \pm 16.3$ \\
Sex & \\
Male & $131(56.2)$ \\
Female & $102(45.8)$ \\
Location & \\
Thoracic & $9(3.9)$ \\
Thoracolumbar & $171(73.4)$ \\
Lumbar & $53(22.7)$ \\
\hline
\end{tabular}

Values are presented as mean \pm standard deviation or number (\%)

Table 3. Comparison of patients types of burst fracture and TLICS score

\begin{tabular}{lcccc}
\hline & \multicolumn{3}{c}{ TLICS } & Total \\
\cline { 2 - 4 } & $\mathbf{1 - 3}$ & $\mathbf{4}$ & $\mathbf{> 5}$ & \\
\hline Stable burst Fx. & $51(21.9)$ & $0(0.0)$ & $0(0.0)$ & $51(21.9)$ \\
Unstable burst Fx. & $21(9.0)$ & $38(16.3)$ & $123(52.8)$ & $182(78.1)$ \\
Total & $72(30.9)$ & $38(16.3)$ & $123(52.8)$ & $233(100.0)$ \\
\hline
\end{tabular}

Values are presented as number (\%). TLICS : thoracolumbar injury classification system, Fx. : fracture

Table 4. Result of 21 patients who diagnosed as unstable thoracolumbar burst fracture, and scored 2 in TLICS

\begin{tabular}{lc}
\hline & Value \\
\hline Body compression $\geq 40 \%$ & $17(80.9)$ \\
Canal compromise $\geq 50 \%$ & $2(9.5)$ \\
Kyphosis $\geq 30^{\circ} \&$ body compression $\geq 40 \%$ & $1(4.8)$ \\
Canal compromise $\geq 50 \%$ \& body compression $\geq 40 \%$ & $1(4.8)$ \\
Total & $21(100.0)$ \\
\hline
\end{tabular}

Values are presented as number (\%). TLICS : thoracolumbar injury classification system

\section{DISCUSSION}

Although many classifications for thoracic and lumbar spinal fractures have been developed, treatment of these fracture remain still controversial. No single method could describe the severity of injury while accounting all neurological, clinical, and radiological characteristics enough to make decision. Numerous studies in the literature have dealt with the stability of the burst fracture. However, the concept of stability, which is the most important factor for the treatment decision-making, is still debated. The concept of burst fracture was first defined by Holdsworth ${ }^{6}$ in 1970. Holdsworth thought the burst fractures to be stable injuries because the anterior and middle columns may be broken down, whereas the posterior usually remains mechanically intact. According to Kelly and Whitesides $^{8)}$, however, complete disruption of the posterior complex can cause the severe vertebral body breakage - the "unstable" burst fracture. Denis ${ }^{4)}$ considered that all thoracolumbar burst fractures would be unstable, insisting that the involvement of the middle column was a sufficient factor for instability without any relation to type or direction of forces acting on the spinal column. Many radiologic parameters such as local kyphotic angle, anterior vertebral height, posterior vertebral height and canal compromise were mentioned to describe the stability ${ }^{2,3,13,16}$. Especially, McAfee et al. ${ }^{11)}$ and McCormack et al. ${ }^{12)}$ emphasized these radiologic morphologies and spinal deformities. However, it is difficult to define the critical values of theses parameters that may be essential for determination of the stability of the thoracolumbar burst fracture. In this concept, we define unstable thoracolumbar burst fracture in case of injury of all three columns, severe canal compromise more than 50\% in axial CT scan of injured level, vertebral height loss more than $40 \%$, kyphosis more than $30^{\circ}$ in plain X-ray and neurologic deficit. Fifty-one patients (21.9\%) who didn't satisfy any of those criteria were diagnosed as stable burst fracture.

TLICS is the first classification system to encounter a neurologic status of the patients. Also, it is easy to utilize. It is based on three major categories : injury morphology, posterior ligamentous complex integrity, and the neurological status. The injury severity score is calculated by summation of each score. A score of $\leq 3$ points suggests nonsurgical treatment, a score of $\geq 5$ suggests surgical treatment, and a score of 4 points suggests either surgical or conservative treatment. Burst frac- 
ture scored 2 in morphology, and 51 patients diagnosed as stable burst fracture showed neither posterior ligamentous complex injury nor neurologic deficit. The TLICS scores of these patients were all 2 which suggest conservative treatment. Although the treatment of thoracolumbar burst fractures remains controversial, many authors believed that conservative treatment for burst fractures without neurological deficit appears to yield acceptable results ${ }^{14)}$. The TLICS score was 2 and the treatment algorithm was perfectly matched in patients with stable thoracolumbar burst fracture. There are also several studies that surgical methods provide immediate spinal stability and more reliably restore the sagittal alignment, as well as vertebral and canal dimensions. In our study, 22 patients (43.1\%) underwent fusion operation in spite of TLICS 2 with stable burst fracture. Twenty-nine (56.9\%) underwent conservative treatment. Seven patients (13.8\%) took percutaneous vertebroplasty or kyphoplasty and 22 patients (43.1\%) took medication, bed-rest and bracing.

Patients who satisfied mentioned criteria were diagnosed as unstable thoracolumbar burst fracture and 182 patients (78.1\%) were included. Twenty-one patients $(9.0 \%)$ who diagnosed as unstable burst fracture scored 2 in TLICS. According to the guideline of Korean Health Insurance Review \& Assessment Service Center, these patients satisfied the unstable thoracolumbar burst fracture and could be an indication of operation. However, in TLICS system, their TLICS score was 2 which suggested conservative treatment. Consequentially, 14 patients (66.7\%) underwent operative treatment with anterior or posterior fusion, four patients (19\%) had conservative treatment with bracing and pain control medication, and three patients (14.3\%) underwent percutaneous vertebroplasty or kyphoplasty.

The TLICS system is thought to be well matched in case of conservative decision-making as all stable thoracolumbar burst fracture patients presented TLICS score 2. However, the controversy of using TLICS to guide surgical treatment has been existed previously. In the study of Joaquim et al. ${ }^{7)}$, the TLICS system matched surgical treatment in only $46.6 \%$ of patients while $100 \%$ matched in conservative decision-making. And all the mismatched patients had a TLICS score of 2 but performed operation. In our study, mismatched patients were found who had TLICS score of 2 but showed unstable thoracolumbar bursting fracture.

There is a disagreement in the international spine surgery community about guidelines for surgical intervention pro- posed by TLICS, rendering evaluation of the reliability of treatment decisions less clinically relevant ${ }^{10}$. According to the TLICS classification, cases which have comminuted burst fracture with intact posterior ligamentous complex and no neurological involvement could score 2. In such cases, the classification algorithm clearly recommended conservative treatment. However, the patients with TLICS score 2 could resulted in further collapse of the vertebral body with marked focal kyphosis, progressive loss of the vertebral body height and deterioration in the pain status. In our study, 21 patients scored 2 in TLICS system, but 14 patients (66.7\%) of them underwent operation. Severe compression of vertebral body height was the most common reason for determining as unstable thoracolumbar burst fracture which leads to operation. The patients satisfied the criteria of unstable burst fracture and underwent operative treatment to prevent progressive vertebral body compression and focal kyphosis. The Korean Health Insurance Review \&Assessment Service Center approved an operation with pedicle screw in those cases. Mattei et al. ${ }^{10)}$ emphasized that patients with comminuted burst fractures deserved special attention, even though initially classified as non-operative according to the TLICS algorithm. He also recommended a close follow-up due to the high likelihood of long-term kyphotic deformity.

Our study is limited by its retrospective nature and involvement of a single center. Although demonstrated reliability, retrospective application of the TLICS cannot decisively prove a safety and efficacy. The decisions for these patients were not based on a TLICS so that we cannot identify how use of the TLICS impacts patients care. Treatment decisions require complete evaluation of the neurological status, however, we only reviewed the medical records of the patients. Thorough evaluation of patients' neurological status was not sufficient. The loss of follow-up among some patients could be a bias in our study, especially patients with conservative treatment group. The progress of kyphosis, signs of late instability could be a complication of conservative treatment.

\section{CONCLUSION}

The TLICS is relatively easy to understand and guide treatment recommendations. It emphasizes very important points of injury (morphology, PLC status, neurology) and could be a 
great help to decide whether to operate or not. But in cases of unstable burst fracture patients, the algorithm of TLICS is insufficient to cover the clinical experiences. Physicians should pay attention in determining treatment plain of thoracolumbar unstable burst fracture patients not to overestimate the TLICS but to be careful for look out other guidelines.

\section{CONFLICTS OF INTEREST}

No potential conflict of interest relevant to this article was reported.

\section{INFORMED CONSENT}

This type of study does not require informed consent.

\section{AUTHOR CONTRIBUTIONS}

\author{
Conceptualization : JHW, HWL, YMK \\ Data curation : JHW, HWL \\ Formal analysis : HWL \\ Methodology: JHW, HWL, HJC, YMK \\ Project administration : YMK \\ Visualization : JHW \\ Writing - original draft : JHW, HWL \\ Writing - review \& editing : JHW, HJC, YMK
}

\section{ORCID}

Jun Hyuk Woo https://orcid.org/0000-0001-7631-2308

Hyun Woo Lee https://orcid.org/0000-0001-8930-2002

Hong June Choi https://orcid.org/0000-0002-7437-2320

Young Min Kwon https://orcid.org/0000-0002-9870-5257

\section{- Acknowledgements}

This paper was supported by the Dong-A University fund.

\section{References}

1. Alpantaki K, Bano A, Pasku D, Mavrogenis AF, Papagelopoulos PJ, Sapkas GS, et al. : Thoracolumbar burst fractures: a systematic review of management. Orthopedics $33:$ 422-429, 2010

2. Cantor JB, Lebwohl NH, Garvey T, Eismont FJ : Nonoperative management of stable thoracolumbar burst fractures with early ambulation and bracing. Spine (Phila Pa 1976) 18 : 971-976, 1993

3. Dai $L Y$, Jiang $S D$, Wang $X Y$, Jiang $L S: A$ review of the management of thoracolumbar burst fractures. Surg Neurol 67 : 221-231; discussion 231, 2007

4. Denis $F$ : The three column spine and its significance in the classification of acute thoracolumbar spinal injuries. Spine (Phila Pa 1976) 8 : 817831, 1983

5. Denis F, Armstrong GW, Searls K, Matta L : Acute thoracolumbar burst fractures in the absence of neurologic deficit. A comparison between operative and nonoperative treatment. Clin Orthop Relat Res (189) : 142-149, 1984

6. Holdsworth F : Fractures, dislocations, and fracture-dislocations of the spine. J Bone Joint Surg Am 52 : 1534-1551, 1970

7. Joaquim AF, Daubs MD, Lawrence BD, Brodke DS, Cendes F, Tedeschi $H$, et al. : Retrospective evaluation of the validity of the thoracolumbar injury classification system in 458 consecutively treated patients. Spine J $13:$ 1760-1765, 2013

8. Kelly RP, Whitesides TE Jr : Treatment of lumbodorsal fracture-dislocations. Ann Surg 167 : 705-717, 1968

9. Magerl F, Aebi M, Gertzbein SD, Harms J, Nazarian S : A comprehensive classification of thoracic and lumbar injuries. Eur Spine J 3 : 184-201, 1994

10. Mattei TA, Hanovnikian J, Dinh DH : Progressive kyphotic deformity in comminuted burst fractures treated non-operatively: the Achilles tendon of the thoracolumbar injury classification and severity score (TLICS). Eur Spine J 23 : 2255-2262, 2014

11. McAfee PC, Yuan HA, Lasda NA : The unstable burst fracture. Spine (Phila Pa 1976) 7 : 365-373, 1982

12. McCormack T, Karaikovic E, Gaines RW : The load sharing classification of spine fractures. Spine (Phila Pa 1976) 19 : 1741-1744, 1994

13. Reid $A B$, Letts RM, Black GB : Pediatric chance fractures: association with intra-abdominal injuries and seatbelt use. J Trauma 30 : 384-391, 1990

14. Stadhouder A, Buskens E, Vergroesen DA, Fidler MW, de Nies F, Oner FC : Nonoperative treatment of thoracic and lumbar spine fractures: a prospective randomized study of different treatment options. J Orthop Trauma 23 : 588-594, 2009

15. Vaccaro AR, Lehman RA Jr, Hurlbert RJ, Anderson PA, Harris M, Hedlund $R$, et al. : A new classification of thoracolumbar injuries: the importance of injury morphology, the integrity of the posterior ligamentous complex, and neurologic status. Spine (Phila Pa 1976) 30 : 2325-2333, 2005

16. Wood K, Buttermann G, Mehbod A, Garvey T, Jhanjee R, Sechriest V : Operative compared with nonoperative treatment of a thoracolumbar burst fracture without neurological deficit. A prospective, randomized study. J Bone Joint Surg Am 85 : 773-781, 2003 